CARDIOVASCULAR MEDICINE

\title{
Mode of death in heart failure: findings from the ATLAS trial
}

\author{
P A Poole-Wilson, B F Uretsky, K Thygesen, J G F Cleland, B M Massie, L Rydén, on \\ behalf of the ATLAS study group
}

Heart 2003;89:42-48

Objective: To investigate markers that predict modes of death in patients with chronic heart failure. Design: Randomised, double blind, three period, comparative, parallel group study (ATLAS, assessment of treatment with lisinopril and survival).

Patients: 3164 patients with mild, moderate, or severe chronic heart failure (New York Heart Association functional class II-IV).

Interventions: High dose (32.5 or $35 \mathrm{mg}$ ) or low dose (2.5 or $5 \mathrm{mg}$ ) lisinopril once daily for a median of 46 months.

Main outcome measures: All cause mortality, cardiovascular mortality, sudden death, and chronic heart failure death related to prognostic factors using competing risks analysis. Mode of death was classified by trialists and by an independent end point committee.

Results: Age, male sex, pre-existing ischaemic heart disease, increasing heart rate, creatinine concentration, and certain drugs taken at randomisation were markers of increased risk of all cause mortality and cardiovascular death. There were risk markers for sudden death that were different from the risk

Accepted 25 July 2002

Correspondence to: Professor Philip A Poole-Wilson, Faculty of Medicine, Imperial

College, Dovehouse Street, London SW3 6LY, UK; p.poole-wilson@ic.ac.uk markers for death from chronic heart failure. Low systolic blood pressure at baseline, raised creatinine, reduced serum sodium or haemoglobin, and increased heart rate were associated with chronic heart failure death. Use of $\beta$ blockers or antiarrhythmic agents (mainly amiodarone) was associated with a reduced risk of sudden death, whereas long acting nitrates and previous use of angiotensin converting enzyme inhibitors were markers for increased risk.

Conclusions: The use of competing risks analysis on the data from the ATLAS study has identified variables associated with certain modes of death in heart failure patients. This approach to analysing outcomes may make it possible to predict which patients might benefit most from particular therapeutic interventions.

C hronic heart failure is associated with a poor prognosis. More than $50 \%$ of patients with New York Heart Association (NYHA) class IV chronic heart failure die within 12 months, and 50\% of all patients with chronic heart failure die within five years of diagnosis. ${ }^{1}$ Although the prognosis has improved in recent years, in clinical practice survival is often worse than seen in trials, with three year survival rates of $38 \%,{ }^{2}$ and in one recent London study, $62 \%$ survival after one year. ${ }^{3}$ Adverse prognostic markers in patients with chronic heart failure include age, severity of heart failure, diabetes mellitus, left ventricular dysfunction, specific aetiologies of heart failure, raised creatinine, relatively lower systemic arterial pressure, neurohormonal activation, and the presence of certain ambient arrhythmias. ${ }^{45}$

There is a difference between the underlying cause of death (for example, pump failure) and the immediate circumstances of death or the mode of death (sudden unexpected death, myocardial infarction related death, etc). Although much is known about the degree of risk associated with each prognostic factor in chronic heart failure, there is less information on the relation between the cause of death and the mode of death. Investigation of the factors associated with different modes of death could be a first step in developing predictors of modes of death in heart failure. If physicians are to be able eventually to use prognostic factors to guide treatment decisions, it is important to differentiate between factors causing increased mortality and factors that are simply markers of a worse prognosis. ${ }^{4}$ The latter typically are markers of disease severity rather than therapeutic targets in their own right. For instance, improving haemodynamic variables-such as the left ventricular ejection fraction-was expected to have a beneficial effect on survival. In controlled trials, this has not been substantiated. Positive inotropes, such as phosphodiesterase inhibitors, increased left ventricular ejection fraction but reduced survival. ${ }^{6}$

Results of the ATLAS (assessment of treatment with lisinopril and survival) clinical trial of the angiotensin converting enzyme (ACE) inhibitor lisinopril have provided detailed data on the circumstances of death in patients with chronic heart failure ${ }^{7-9}$ We have been able to identify which deaths were from cardiovascular causes, and, within that group, which deaths were classified as sudden unexpected deaths and which were caused by progressive chronic heart failure.

Competing risks analysis is an established statistical technique which is appropriate when the intention is to distinguish between multiple prognostic indicators. ${ }^{10}{ }^{11}$ Therefore, competing risks analysis has been undertaken on the ATLAS database to identify potential prognostic factors, including the dose of lisinopril, and to investigate the contributing hazard or risk of each of these factors for each mode of death. It was anticipated that some factors would have a link with particular modes of death, and that patterns of factors might be recognisable that could be used to predict the likely mode of cardiovascular death in different patient groups.

Abbreviations: AIRE, acute infarction ramipril efficacy; ATLAS, assessment of treatment with lisinopril and survival; NYHA, New York Heart Association; SOLVD, studies of left ventricular dysfunction; V-HeFT II, vasodilator heart failure trial II 
Table 1 Demographic characteristics and mean levels of covariates used in the analysis of patients in the ATLAS trial by high dose (32.5 or $35 \mathrm{mg}$ ) and low dose $(2.5$ or $5 \mathrm{mg}$ ) lisinopril

\begin{tabular}{|c|c|c|}
\hline Demographic characteristics and mean levels of covariates & High dose lisinopril $(n=1568)$ & Low dose lisinopril $(n=1596)$ \\
\hline Age (years) (mean (SD)) & $63.6(10.5)$ & $63.6(10.3)$ \\
\hline Age $\geqslant 70$ years & $513(32.7)$ & $475(29.8)$ \\
\hline Male sex & $1251(79.8)$ & $1265(79.3)$ \\
\hline \multicolumn{3}{|l|}{ Race } \\
\hline White & $1417(90.4)$ & $1451(90.9)$ \\
\hline Afro-Caribbean & $99(6.3)$ & $105(6.6)$ \\
\hline Other* & $52(3.3)$ & $40(2.5)$ \\
\hline \multicolumn{3}{|l|}{ Aetiology of chronic heart failure $\dagger$} \\
\hline Ischaemic heart disease & $999(63.7)$ & $1036(64.9)$ \\
\hline Hypertension & $301(19.2)$ & $332(20.8)$ \\
\hline Idiopathic dilated cardiomyopathy & $453(28.9)$ & $437(27.4)$ \\
\hline Valvar disease & $96(6.1)$ & $88(5.5)$ \\
\hline Aetiology unknown/not specified & $43(2.7)$ & $43(2.7)$ \\
\hline Other cause & $77(4.9)$ & $66(4.1)$ \\
\hline \multicolumn{3}{|l|}{ NYHA class } \\
\hline ॥ & $262(16.7)$ & $231(14.5)$ \\
\hline III & $1194(76.1)$ & $1252(78.4)$ \\
\hline IV & $112(7.1)$ & $113(7.1)$ \\
\hline LVEF (\%) (mean (SD)) & $22.6(5.7)$ & $22.6(5.6)$ \\
\hline Weight (kg) (mean (SD)) & $78.9(16.0)$ & $78.9(16.7)$ \\
\hline \multicolumn{3}{|l|}{ Previous ACE inhibitor } \\
\hline Yes & $1390(88.6)$ & $1420(89.0)$ \\
\hline \multicolumn{3}{|l|}{ Previous myocardial infarction } \\
\hline \multirow{2}{*}{\multicolumn{3}{|c|}{ Severe or unstable angina pectoris }} \\
\hline & & \\
\hline Yes & $226(14.4)$ & $250(15.7)$ \\
\hline \multicolumn{3}{|l|}{ Atrial fibrillation } \\
\hline Yes & $286(18.2)$ & $276(17.3)$ \\
\hline Duration of heart failure (years) (mean (SD)) & $3.4(3.5)$ & $3.4(3.5)$ \\
\hline Heart rate (beats/min) (mean (SD)) & $80(14)^{\circ}$ & $80(15)^{b}$ \\
\hline \multicolumn{3}{|l|}{ Blood chemistry/haematology } \\
\hline Sodium (mmol/l) (mean (SD)) & $139.4(3.8)^{\mathrm{c}}$ & $139.5(3.6)^{\mathrm{d}}$ \\
\hline Potassium (mmol/l) (mean (SD)) & $4.5(0.5)^{\mathrm{e}}$ & $4.5(0.5)^{f}$ \\
\hline Creatinine $(\mu \mathrm{mol} / \mathrm{l})$ (mean (SD)) & $119.7(31.9)^{\mathrm{g}}$ & $121.3(33.1)^{\mathrm{h}}$ \\
\hline Haemoglobin (g/l) (mean (SD)) & $143(15)^{i}$ & $143(15)^{i}$ \\
\hline \multicolumn{3}{|c|}{ 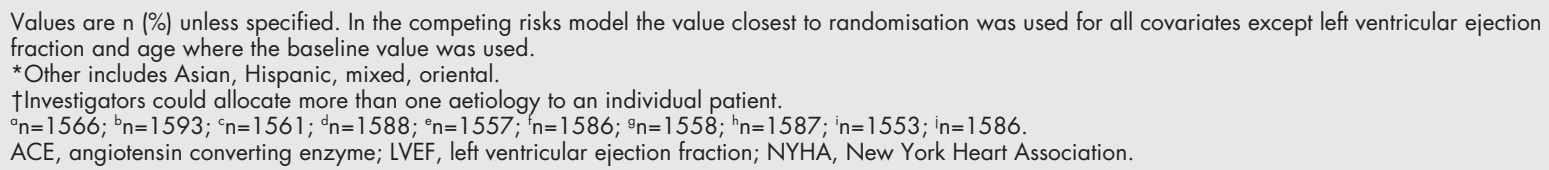 } \\
\hline
\end{tabular}

\section{METHODS}

Details of the trial design ${ }^{7}$ and the main results ${ }^{8}$ of the ATLAS study have been published.

\section{Study design and treatments}

ATLAS was an international multicentre trial of lisinopril in patients with chronic heart failure. A short open label tolerability period was followed by a double blind dose titration period and a double blind treatment maintenance period. In all, 3164 patients with mild, moderate, or severe chronic heart failure (NYHA class II-IV) were randomised to once daily treatment with either high dose (32.5 or $35 \mathrm{mg}$ ) or low dose (2.5 or $5 \mathrm{mg}$ ) lisinopril (Zestril; AstraZeneca, Macclesfield, UK). The trial was conducted between October 1992 and September 1997. The follow up period was 39-58 months, with a median of 46 months. With the exception of other ACE inhibitors, concomitant drug treatment was permitted throughout the active treatment period. The primary trial end point was all cause mortality. Although several secondary end points were prespecified, the major secondary end point was combined all cause mortality and all cause hospital admission. Mode of death and reasons for hospital admission were documented by the investigator, and the mode and cause of death were adjudicated by the end point committee.

\section{Study population}

The demographics and baseline patient characteristics for the ATLAS trial are summarised in table 1 . The treatment groups were well balanced for the variables used in the competing risks analysis. Most patients had moderately severe congestive heart failure, with 77\% in NYHA class III. The mean ejection fraction was $22.6 \%$. Approximately $89 \%$ of the patients had previously received treatment with an ACE inhibitor.

\section{Methods of end point committee}

Deaths during the trial were adjudicated by a two member end point committee. Relevant information was collected on end point event forms and supporting documentation was sent simultaneously to the two end point committee members without details of the randomised treatment (fig 1). The supporting documentation included copies of case record forms, necropsy reports, death certificates, hospital notes, and 12 lead ECG recordings. If the committee members disagreed about the mode of death, the case was discussed at a meeting and further information could be requested from the investigator. If agreement was still not reached, the death was recorded as unclassifiable.

Deaths were classified as either cardiovascular or noncardiovascular. Cardiovascular deaths were subdivided into sudden unexpected death, heart failure death, myocardial infarction related death, and other cardiovascular deaths (which included pulmonary embolism, peripheral thromboembolism, stroke, deaths related to a vascular procedure, or major cardiovascular event not otherwise specified). Sudden death included observed arrhythmic deaths and sudden deaths not attributable to intractable myocardial infarction or any other identifiable cause. These deaths were also recorded as "witnessed" or "unwitnessed". Patients with sudden loss of 


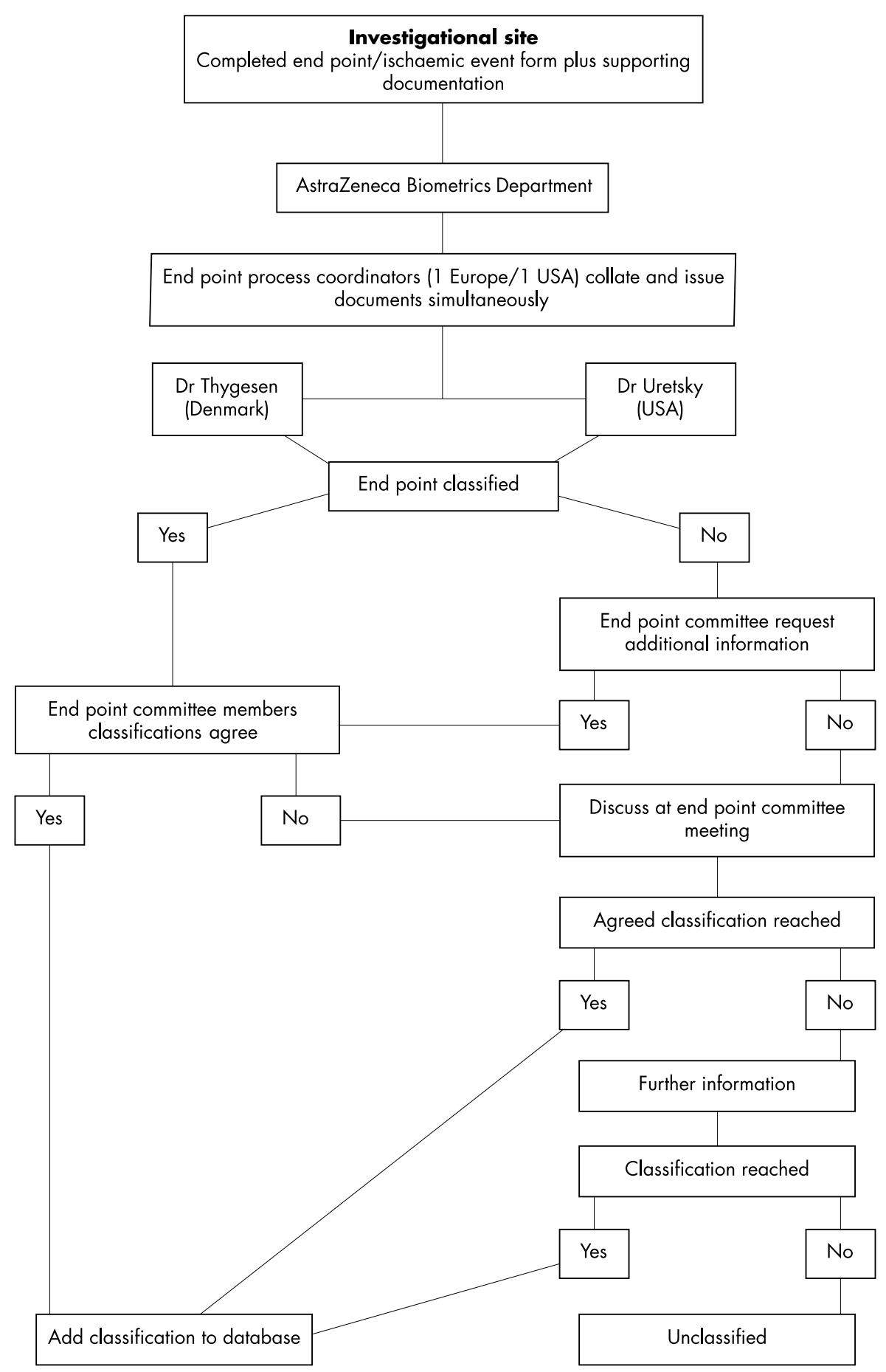

Figure 1 Flow diagram showing the review process of the end point committee involved in deciding the mode of death for each patient in the ATLAS trial.

consciousness who were resuscitated but later died were described as having a sudden death. Non-cardiovascular death and other causes of cardiovascular death, such as pulmonary embolism and myocardial infarction related death, were not analysed separately because of the small numbers in each group.

Death from chronic heart failure included patients with intractable heart failure for the purposes of this study, even if the terminal event was an arrhythmia.'

There is a high correlation between out of hospital death and death classified as sudden cardiac death by end point committees. ${ }^{12}{ }^{13}$ Inpatient deaths represent a more mixed picture of sudden and progressive heart failure death. Accordingly, out of hospital death was used as an objective surrogate marker of sudden cardiac death. ${ }^{14}{ }^{15}$ The exception to this was in the small number of cases where a patient with advanced heart failure chose to remain at home to die; these out of hospital deaths were, in the main, from heart failure and were classified separately. Transplantation was classified as cardiovascular death from chronic heart failure. Transplant details were declared by the investigator but not classified by the end point committee.

Confirmatory competing risks analyses were based on two complementary, objective classifications of death and compared for end point committee designated sudden cardiac death and out of hospital death.

\section{Measurement and classification of data}

The following groups of measurements were used to characterise patients for the competing risks analysis of mode 
Table 2 Contingency table of end point committee decisions for classification of mode of death by the number of patients who died in hospital

\begin{tabular}{lllc}
\hline $\begin{array}{l}\text { End point committee } \\
\text { classification of mode of } \\
\text { death }\end{array}$ & $\begin{array}{l}\text { In-hospital } \\
\text { death }\end{array}$ & $\begin{array}{l}\text { Out of } \\
\text { hospital } \\
\text { death }\end{array}$ & $\begin{array}{l}\text { Not } \\
\text { reported }\end{array}$ \\
\hline Sudden unexpected & 149 & 439 & 1 \\
Chronic heart failure & 309 & 97 & 39 \\
Other deaths & 271 & 71 & 7 \\
\hline
\end{tabular}

of death: patient characteristics (age, sex, history of ischaemic heart disease), haemodynamics (left ventricular ejection fraction, NYHA class (classes III and IV were combined), systolic blood pressure, diastolic blood pressure, and heart rate), laboratory variables (serum sodium, potassium, creatinine, and haemoglobin), and drugs being taken at randomisation (antidiabetic agents, aspirin, $\beta$ blockers, previous ACE inhibitors, long acting nitrates, short acting nitrates, antiarrhythmic agents, calcium channel blockers, and anticoagulants/ warfarin). The antiarrhythmic drugs taken (by $9.7 \%$ of patients at randomisation) included amiodarone $(93 \%$ of patients receiving antiarrhythmics), aprindine, bretylium tosilate, disopyramide, flecainide, propafenone, and tocainide.

The data collected were either continuous (age, left ventricular ejection fraction, blood pressure, clinical chemistry) or discrete (sex, history of ischaemia, drugs at randomisation). Mean levels of the covariates used in the competing risks analysis are shown in table 1 .

\section{Competing risks analysis}

Competing risks analysis is an appropriate technique when it is of interest to distinguish between different modes of death and treat them separately in a model. Each mode of death is looked at in turn and all other modes of death are censored in the Cox proportional hazards model, using multivariate analysis. This is appropriate because a patient dying from progressive heart failure, for example, is no longer at risk for any other mode of death. This type of analysis can distinguish the effect of baseline covariates both within and between different modes of death. Multivariate analysis was used to adjust for prognostic factors, but within a category, factors were not adjusted owing to the potential correlation (for example, drugs at randomisation were not adjusted for other drugs at randomisation).

\section{RESULTS}

\section{Classification of cardiovascular deaths}

There were 1383 deaths in the study group: 1224 cardiovascular deaths, 146 non-cardiovascular deaths, and 13 deaths which could not be classified owing to lack of information. The end point committee reached the following conclusions on the mode of cardiovascular deaths: 589 sudden deaths, 445 chronic heart failure deaths (including 39 transplants), 97 myocardial infarction related deaths, and 93 other cardiovascular deaths. There were 729 deaths in hospital, of which 309 were from chronic heart failure and 149 were sudden, and 607 out of hospital deaths, of which 97 were from chronic heart failure and 439 were sudden. In 47 cases the location of death was not reported. In-hospital and out of hospital deaths, and sudden versus chronic heart failure deaths, are shown in table 2 .

\section{Interpretation of competing risks analysis table for mode of death}

The results of the competing risks analysis for mode of death in the ATLAS study are shown in table 3, where hazard ratios of different prognostic factors for each mode of death are given. The data can be interpreted both horizontally (to show the effect of individual indicators on each mode of death) and vertically (to establish a pattern of indicators that may be associated with a certain mode of death).

It should be noted that "all cardiovascular mortality" is a subset of "all cause mortality" and that both "sudden death" and "chronic heart failure death" are subsets of "all cardiovascular mortality". The hazard ratio for a continuous covariate can be interpreted by calculating the estimated percentage change for each unit increase in the covariate. For hazard ratios $>1$, a unit increase in the covariate will increase the risk of the event. For hazard ratios $<1$, a unit increase in the covariate will decrease the risk of the event. For example, for systolic blood pressure the hazard ratio is 0.996 for all cause mortality. The percentage change is $(1 /-0.996) \times 100=-0.4$. Therefore, for each $1 \mathrm{~mm} \mathrm{Hg}$ increase in systolic blood pressure the hazard of death goes down by an estimated $0.4 \%$.

Unless stated otherwise, all results mentioned in the text are significant.

Table 3 shows whether certain hazard ratios were statistically significant, but does not imply statistical differences between the various predictive factors. The results illustrate a group of factors strongly associated with different modes of death, but make no inference about differences between individual factors.

\section{All cause mortality}

Increased mortality was related to increasing age, male sex, the presence of ischaemic heart disease, increasing heart rate, increasing plasma creatinine, and long acting nitrates or ACE inhibitors at randomisation. The risk of death from any cause was lower in patients with a higher ejection fraction, a higher systolic blood pressure at baseline, and those taking aspirin or $\beta$ blockers at randomisation.

\section{Markers associated with an increased risk of sudden death}

The risk of sudden death increased with increasing age, the presence of ischaemic heart disease, increasing plasma creatinine, and in those taking long acting nitrates or ACE inhibitors at randomisation. There was also a trend towards a higher risk with male sex but this did not reach significance.

The risk of sudden death was lower in patients with a high ejection fraction and those taking $\beta$ blockers or antiarrhythmic agents at randomisation.

\section{Markers associated with an increased risk of death from heart failure}

There was an increased risk of death from chronic heart failure with increasing age, with the presence of ischaemic heart disease, and in patients with more severe chronic heart failure and neurohormonal activation at baseline-for example, in those patients with decreased left ventricular ejection fraction, increased NYHA score (not significant), and increased heart rate. Decreased serum sodium, which may reflect the use of higher doses of diuretics, increased serum creatinine, and decreased haemoglobin were associated with increased risk. The use of antiarrhythmic drugs was related to an increased risk of chronic heart failure death.

\section{Comparison of markers of increased risk of sudden death and death from chronic heart failure}

Several markers were associated with increased risk of both sudden and chronic heart failure death-for example, age, increasing serum creatinine, and the presence of ischaemic heart disease; however, all reached a greater level of significance for death from heart failure than for sudden death.

Increased heart rate was associated with increased risk of death from chronic heart failure but not of sudden death. 
Table 3 Competing risks analysis hazard ratios and level of significance for hazard ratios of prognostic factors for all cause mortality, all cardiovascular mortality, sudden death, and chronic heart failure death

\begin{tabular}{|c|c|c|c|c|c|}
\hline \multirow[b]{2}{*}{ Prognostic factor } & \multicolumn{5}{|l|}{ Hazard ratio } \\
\hline & $\begin{array}{l}\text { All cause mortality } \\
(\mathrm{H}=666, \mathrm{~L}=717)\end{array}$ & $\begin{array}{l}\text { All cardiovascular } \\
\text { mortality } \\
(\mathrm{H}=583, \mathrm{~L}=641)\end{array}$ & $\begin{array}{l}\text { CHF death } \\
(\mathrm{H}=203, \\
\mathrm{L}=242)\end{array}$ & $\begin{array}{l}\text { Sudden death } \\
(H=286, \\
L=303)\end{array}$ & $\begin{array}{l}\text { Out of hospital } \\
\text { death } \\
(\mathrm{H}=285, \mathrm{~L}=322)\end{array}$ \\
\hline Lisinopril treatment (high: low) & 0.923 & 0.903 & 0.827 & 0.922 & 0.879 \\
\hline \multicolumn{6}{|l|}{ Demographics } \\
\hline Age (years) - continuous & $1.025^{* * *}$ & $1.022 * * *$ & $1.029 * * *$ & $1.012^{* *}$ & $1.019 * * *$ \\
\hline Sex (male: female) & $1.164^{*}$ & 1.156 & 1.095 & 1.232 & $1.349 *$ \\
\hline Ischaemic heart disease (yes/no) & $1.315^{* * *}$ & $1.393^{* * *}$ & $1.466 * *$ & $1.251^{*}$ & 1.214 \\
\hline \multicolumn{6}{|l|}{ Haemodynamics } \\
\hline LVEF $(\%)$ - continuous & $0.973 * * *$ & $0.969 * * *$ & $0.960 * * *$ & $0.970 * * *$ & $0.974 * * *$ \\
\hline NYHA (IIII + IV : II) & 1.106 & 1.110 & 1.301 & 1.046 & 0.982 \\
\hline Systolic BP (mm Hg) - continuous & $0.996 *$ & $0.995^{*}$ & $0.989 * *$ & 0.998 & 0.997 \\
\hline Diastolic BP (mm Hg) - continuous & 0.995 & 0.997 & 0.989 & 0.999 & 1.000 \\
\hline Heart rate (beats $/ \mathrm{min}$ ) - continuous & $1.011 * * *$ & $1.012^{* * *}$ & $1.025^{* * *}$ & 1.000 & 1.006 \\
\hline \multicolumn{6}{|l|}{ Laboratory values } \\
\hline Sodium (mmol/l) - continuous & 0.987 & 0.987 & $0.974^{*}$ & 0.994 & 1.002 \\
\hline Potassium $(\mathrm{mmol} / \mathrm{l})$ - continuous & 0.923 & 0.910 & 1.009 & 0.865 & 0.933 \\
\hline Creatinine $(\mu \mathrm{mol} / \mathrm{l})$ - continuous & $1.005^{* * *}$ & $1.004^{* * *}$ & $1.006 * * *$ & $1.003^{*}$ & 1.004 ** \\
\hline Haemoglobin $(\mathrm{g} / \mathrm{l})$ - continuous & 0.983 & 0.999 & $0.927^{*}$ & 1.036 & 0.983 \\
\hline \multicolumn{6}{|l|}{ Drugs at randomisation } \\
\hline Antidiabetic (yes 611: no 2553) & 1.093 & 1.101 & 1.180 & 1.143 & 1.137 \\
\hline Aspirin (yes 1278: no 1886) & $0.825^{* *}$ & $0.859 *$ & $0.704 * *$ & 1.031 & 0.975 \\
\hline$\beta$ Blockers (yes 353: no 2811) & $0.792^{*}$ & $0.769^{*}$ & 0.781 & $0.714^{*}$ & $0.637^{* *}$ \\
\hline Long acting nitrates (yes 786: no 2378) & $1.241^{* * *}$ & $1.289 * \star *$ & 1.165 & $1.351^{* *}$ & $1.283^{* *}$ \\
\hline Short acting nitrates (yes 726 : no 2438) & 1.087 & 1.120 & 1.171 & 1.158 & 1.026 \\
\hline Previous ACE inhibitor (yes 2810: no 354) & $1.271^{* *}$ & $1.310 * *$ & 1.369 & $1.337^{*}$ & 1.176 \\
\hline Antiarrhythmics (yes 306: no 2858)† & 1.010 & 1.018 & $1.457^{*}$ & $0.703 *$ & 0.627 ** \\
\hline Calcium channel blockers (yes 369: no 2795) & 1.027 & 0.986 & 0.875 & 0.984 & 1.109 \\
\hline Anticoagulants/warfarin (yes 1126: no 2038) & 0.999 & 1.040 & 0.978 & 1.187 & 1.107 \\
\hline \multicolumn{6}{|c|}{$\begin{array}{l}\text { *Represents a significant hazard ratio }(1: 0) \text { at } p<0.05 \text {. } \\
* * \text { Represents a significant hazard ratio }(1: 0) \text { at } p<0.001 \text {. } \\
* * * \text { Represents a significant hazard ratio }(1: 0) \text { at } p<0.0001 \text {. } \\
\dagger \text { Antiarrhythmic drugs were received by } 9.7 \% \text { of patients at randomisation and included amiodarone }(93 \% \text { of patients receiving antiarrhythmics), } \\
\text { aprindine, bretylium tosilate, disopyramide, flecainide, propafenone, and tocainide. } \\
\text { ACE, angiotensin converting enzyme; BP, blood pressure; CHF, chronic heart failure; } \mathrm{H} \text {, high dose lisinopril; L, low dose lisinopril; LVEF, left ventricular } \\
\text { ejection fraction; NYHA, New York Heart Association. }\end{array}$} \\
\hline
\end{tabular}

Increased systolic blood pressure, serum sodium, and haemoglobin were associated with a lower risk of both chronic heart failure death and sudden death; however, these variables were significant only for reduced risk of chronic heart failure death.

Drug use at randomisation was associated with different modes of death. The use of long acting nitrates or ACE inhibitors at randomisation was associated with an increased risk of sudden death but not of chronic heart failure death. The use of antiarrhythmic agents was associated with an increased risk of chronic heart failure death and a reduced risk of sudden death (amiodarone was used in $93 \%$ of cases).

The use of aspirin was associated with a reduced risk of chronic heart failure death but had no effect on the risk of sudden death. The use of $\beta$ blockers was associated with a reduced risk of sudden death but not of chronic heart failure death.

\section{Similarities between risk markers}

"All cardiovascular mortality" is a combination mainly of sudden death and chronic heart failure death, with a small contribution from "other cardiovascular deaths". "All cause mortality" is a combination of "all cardiovascular deaths" with a small contribution from "non-cardiovascular deaths". Thus the factors that will influence both "all cardiovascular deaths" and "all cause mortality" will predominantly be those influencing sudden death and chronic heart failure death in the same direction. These factors included increasing age, history of previous ischaemic heart disease, decreased left ventricular ejection fraction, and increased serum creatinine.

\section{Objectivity of end point committee decisions}

The classification of in-hospital or out of hospital deaths from the ATLAS trial shows that most sudden deaths were out of hospital, whereas deaths from chronic heart failure were usually in hospital (table 2). Table 3 shows that out of hospital deaths were associated with similar factors to sudden death. Prognostic indicators for out of hospital deaths (as classified by ATLAS clinical report forms) and sudden death (as classified by the end point committee) correlated in 19 of 22 factors in the competing risks analysis (table 3 ). These data suggest that the end point committee assessment was objective.

\section{DISCUSSION}

In the ATLAS study a high dose ( 32.5 or $35 \mathrm{mg}$ once daily) of the ACE inhibitor lisinopril, compared with a low dose (2.5 or $5 \mathrm{mg}$ once daily), reduced the combined end points of "all cause mortality and all cause hospital admissions" $(p=0.002)$ as well as "all cause mortality and hospital admission for heart failure" $(p<0.001){ }^{8}$ The high dose of lisinopril was well tolerated. ${ }^{8}{ }^{16}$ The analysis reported in this paper has identified factors associated with all cause mortality, and with death classified as "sudden" or as a result of progressive heart failure. Although these associations result from observational rather than randomised evidence, they provide a useful starting point for the development of prognostic indices for mode of death. If the mode of death could be predicted from a single subset of clinical variables, 
then in the future specific treatments could be tested in identifiable subgroups of patients with heart failure who are at risk of certain modes of death.

As a proportion of all deaths classified, sudden death made up $48 \%$ of deaths in the ATLAS study, compared with $32-34 \%$ in other studies of ACE inhibitors in chronic heart failure. ${ }^{14}$ This difference may reflect the classification system used by the end point committee in the ATLAS study, or differences in the aetiology of heart failure or the populations studied. ${ }^{16}{ }^{17}$ Sudden death is notoriously difficult to define, but is usually thought of as a death attributable to an opportunistic arrhythmia.

The mode of death should be distinguished from the cause of death, ${ }^{14}{ }^{18}$ and such an analysis has been conducted in this study. In some studies the classification of mode of death, especially sudden death, has been inconsistent and discordant between observers. ${ }^{19}$ In the ATLAS study, classification of mode of death was undertaken by an independent two member end point committee which reviewed the data for all patients and determined the mode of death; this allowed greater consistency.

The factors associated with cardiovascular death were similar to those associated with death from all causes (all cause mortality), because cardiovascular deaths made up the majority of all deaths.

Competing risks analysis shows that the factors associated with particular modes of death include those indicative of the extent of damage to heart muscle (left ventricular ejection fraction) and the body's metabolic response to diminished cardiac function (sodium and creatinine concentrations), heart rate, and certain previous drugs treatments.

Death from chronic heart failure in the ATLAS study was associated with an overlapping but slightly different subset of predictive factors compared with sudden death; ischaemic heart disease was associated with both modes of death. In general, death associated with progressive chronic heart failure was linked with the symptoms and features of worsening heart failure (NYHA classification, tachycardia, reduced renal function, low ejection fraction), whereas sudden death was associated with a low ejection fraction and treatment with long acting nitrates or ACE inhibitors. Antiarrhythmic treatment (not including $\beta$ blockers) was associated with a reduced risk of sudden death but an increased risk of death from chronic heart failure. In previous studies, antiarrhythmic agents increased death rates. ${ }^{20}$ For this reason, flecainide and encainide were not included in this study protocol, although a small number of patients received these agents. Amiodarone reduces mortality from sudden death in chronic heart failure, and, unlike other agents, does not increase overall mortality. ${ }^{21}$

There is controversy over the use of aspirin in heart failure. Aspirin and ACE inhibitors may have opposing effects on prostaglandins and therefore may interact negatively. ${ }^{22}$ Aspirin also attenuates the effects of ACE inhibitors in heart failure..$^{23}$ Some studies on the use of aspirin in heart failure have shown a deleterious and some a beneficial effect. ${ }^{25-28}$

In the ATLAS study, aspirin use was associated with a reduced risk of all cause mortality, cardiovascular mortality, and death from chronic heart failure, but not a reduced risk of sudden death. This effect was observed in both the high dose and the low dose lisinopril groups, and there is no evidence to suggest that comparisons between the high and low dose groups were affected by whether the patients were taking aspirin.

High dose lisinopril produced a trend to improved survival ${ }^{8}$ and was associated with a reduced risk for all modes of death in this analysis (table 3), although significance was not achieved. These data are supported by safety and tolerability data, which show that high dose lisinopril was as well tolerated as low dose lisinopril, ${ }^{16}$ even in high risk subgroups such as diabetic patients and the elderly. ${ }^{29}$

The technique of competing risks analysis has been used previously in studies of surgical treatment of coronary disease. ${ }^{11}$ It has also been used in a small study of bisoprolol in which a subgroup of patients with advanced heart failure was shown to gain more benefit from treatment than other patients. ${ }^{30}$ This is the first large chronic heart failure study in which the technique has been used. As with any statistical technique, the advantages and limitations of the methodology have to be considered in evaluating the data analysed. In performing the competing risks analysis many subanalyses of the data were undertaken to evaluate the numerous variables identified in table 3, increasing the chance of a significant $p$ value. In this paper we have identified trends as well as significant values in recognition of the fact that with many subanalyses, significance is less powerful. In addition, the different numbers of patients in each group will affect the significance of the results.

While this analysis provides valuable information on associations between risk factors and mode of death, the trial design does not permit any inferences about causal relations between risk factors and mode of death. However, the statistical associations allow the description of sets of clinical characteristics linked, causally or otherwise, with given patient outcomes. The apparent increase in mortality risk with age is unsurprising, and the association of low systolic blood pressure and chronic heart failure death confirms previous reports. ${ }^{31}$ In addition, the association of ischaemic heart disease with both chronic heart failure death and sudden death tends to confirm the results of Uretsky and colleagues, in which unrecognised myocardial infarction was often found to precede both modes of death. ${ }^{9}$

The data from this analysis of the ATLAS study have implications for the choice of treatment of patients with chronic heart failure and also for the development of new treatments for this condition. Identification of subgroups of patients whose mode of death can be predicted may allow improved medical management, although in the case of sudden death it may be necessary to subdivide the patients further into those in whom the cause is primarily electrical and those in whom vascular disease is the most important factor. ${ }^{17}$ The wider use of competing risks analysis is beyond the scope of this paper, but the analysis reported here may provide the basis for the development of computer based predictive models to estimate an individual patient's risk of a particular mode of death. ${ }^{30}$

\section{Conclusions}

The use of competing risks analysis on the data from the ATLAS study has identified variables associated with certain modes of death in patients with heart failure. While there is quite an overlap in predictive markers, in general death from progressive heart failure is associated with several markers of more severe left ventricular dysfunction and neurohormonal activation, as well as ischaemic heart disease, whereas sudden death is most closely related to markers of ischaemic heart disease. This approach to analysing outcomes may in the future enable us to predict which patients might benefit most from particular therapeutic interventions.

\section{ACKNOWLEDGEMENTS}

Supported by grants from AstraZeneca, Alderley Park, Macclesfield, UK. We would like to thank all the clinical sites and investigators for their participation in this trial. A full list of the investigators participating in the study has been published by Packer et al in Circulation.

\footnotetext{
Authors' affiliations

P A Poole-Wilson, Faculty of Medicial Imperial College, London, UK

B F Uretsky, University of Texas, Galveston, Texas, USA

K Thygesen, Aarhus University Hospital, Aarhus, Denmark

J G F Cleland, University of Hull, Hull, Humberside, UK

B M Massie, University of California, San Francisco, California, USA

L Rydén, Karolinska Institute, Stockholm, Sweden
} 


\section{REFERENCES}

1 Cowie MR, Mosterd A, Wood DA, et al. The epidemiology of heart failure. Eur Heart J 1997; 18:208-25.

2 Cleland JGF, Gemmell I, Khand A, et al. Is the prognosis of heart failure improving? Eur J Heart Fail 1999;1:229-41.

3 Cowie MR, Wood DA, Coats A, et al. Survival of patients with a new diagnosis of heart failure: a population based study. Heart 2000;83:505-10.

4 Coats AJS, Poole-Wilson PA. The syndrome of heart failure. In: Weatherall DJ, Ledingham JGG, Warrell DA eds. Oxford textbook of medicine. Oxford: Oxford University Press, 1996:2228-38.

5 Cowburn PJ, Cleland JG, Coats AJ, et al. Risk stratification in chronic heart failure. Eur Heart J 1998;19:696-710.

6 Packer M, Carver JR, Rodeheffer RJ, et al. Effect of oral milrinone on mortality in severe chronic heart failure. The PROMISE study research group. N Engl J Med 1991;325:1468-75.

7 Cleland JGF, Armstrong JD, Horowitz JD, et al. Baseline clinical characteristics of patients recruited in the assessment of treatment with lisinopril and survival study. Eur J Heart Fail 1999;1:73-9.

8 Packer M, Poole-Wilson PA, Armstrong PW, et al. Comparative effects of low doses and high doses of the angiotensin converting-enzyme inhibitor, lisinopril, on morbidity and mortality in chronic heart failure. Circulation 1999:100:2312-18.

9 Uretsky BF, Thygesen K, Armstrong PW, et al. Acute coronary findings at autopsy in heart failure patients with sudden death. Results from the assessment of treatment with lisinopril and survival (ATLAS) trial. Circulation 2000;102:61 1-16.

10 Moeschberger ML, Klein JP. Statistical methods for dependent competing risks. Lifetime Data Analysis 1995;1:195-204.

11 Risum Ø, Abdelnoor RM, Svennevig JL, et al. Risk factors for early and late mortality in surgical treatment of coronary artery disease. Cardiovasc Surg 1995; 3:537-44.

12 The Acute Infarction Ramipril Efficacy (AIRE) Study Investigators. Effect of ramipril on mortality and morbidity of survivors of acute myocardial infarction with clinical evidence of heart failure. Lancet 1993;342:821-8.

13 Epstein AE, Powell J, Yao Q, et al. In-hospital versus out-of-hospital presentation of life-threatening ventricular arrhythmias predicts survival: results from the AVID registry. Antiarrhythmics versus implantable defibrillators. J Am Coll Cardiol 1999;34:1111-16.

14 Narang R, Cleland JGF, Erhardt L, et al. Mode of death in chronic heart failure. A request and proposition for more accurate classification. Eur Heart J 1996:17:1390-403.

15 Cleland JGF, Erhardt L, Murray G, et al. Effect of ramipril on morbidity and mode of death among survivors of acute myocardial infarction with clinical evidence of heart failure. Eur Heart J 1997;18:41-51.

16 Massie BM, Armstrong PW, Cleland JGF, et al. High doses of angiotensin converting enzyme inhibitors are well-tolerated in patients with chronic heart failure: results from the ATLAS trial. Arch Intern Med $2001 ; 161: 165-71$
17 Cleland JGF, Massie BM, Packer M. Sudden death in heart failure: vascular or electrical? Eur Heart J 1999;1:41-5.

18 Cleland JGF. The clinical course of heart failure and its modification by ACE inhibitors: insights from recent clinical trials. Eur Heart J 1994:15:125-30.

19 Ziesche S, Rector TS, Cohn JN. Interobserver discordance in the classification of mechanisms of death in studies of heart failure. J Card Fail 1995; 1:127-32.

20 Naccarelli GV, Dougherty AH, Wolbrette D, et al. A critical appraisal of the cardiac arrhythmia suppression trial (CAST). Appl Cardiopulm Pathphysiol 1999;4:9-16

21 Pinto JVJ, Ramani K, Neelagaru S, et al. Amiodarone therapy in chronic heart failure and myocardial infarction: a review of the mortality trials with special attention to STAT-CHF and the GESICA trials. Grupo de Estudio de la Sobrevida en la Insuficiencia Cardiaca en Argentina. Prog Cardiovasc Dis 1997;40:85-93.

22 Hall $\mathbf{D}$. The aspirin-angiotensin-converting enzyme inhibitor tradeoff: to halve and halve not. J Am Coll Cardiol 2000;35:1808-12.

23 Guazzi M, Pontone G, Agostoni P. Aspirin worsens exercise performance and pulmonary gas exchange in patients with heart failure who are taking angiotensin-converting enzyme inhibitors. Am Heart J $1999 ; 138: 254-60$.

24 Song KH, Fedyk R, Hoover R. Interaction of ACE inhibitors and aspirin in patients with congestive heart failure. Ann Pharmacother 1999;33:375-7.

25 Witte K, Thackray S, Clark AL, et al. Clinical trials update. IMPROVEMENT-HF, COPERNICUS, MUSTIC, ASPECT-II, APRICOT. Eur J Heart Failure 2000;2:455-60.

26 Jones CG, Cleland JGF. Meeting report - the LIDO, HOPE, MOXCON and WASH studies. Eur J Heart Failure 1999:1:425-31.

27 Flather MD, Yusuf S, Køber L, et al. Long-term ACE-inhibitor therapy in patients with heart failure or left ventricular dysfunction: a systemic overview of data from individual patients. Lancet 2000;355:1575-81.

28 Leor J, Reicher-Reiss H, Goldbourt U, et al. Aspirin and mortality in patients treated with angiotensin-converting enzyme inhibitors: a cohort study of 1 1,575 patients with coronary artery disease. J Am Coll Cardio 1999;33:1920-5.

29 Rydén L, Armstrong PW, Cleland JGF, et al. Efficacy and safety of high-dose lisinopril in chronic heart failure patients at high cardiovascular risk, including those with diabetes mellitus. Results from the ATLAS trial. Eur Heart J 2000;21:1967-78

30 Funck-Brentano C, Lancar R, Le Heuzey J-Y, et al. Predictors of medical events in patients enrolled in the cardiac insufficiency bisoprolol study (CIBIS): a study of the interactions between $\beta$-blocker therapy and occurrence of critical events using analysis of competitive risks. Am Heart J 2000;139:262-71.

31 Robbins $M$, Francis G, Pashkow FJ, et al. Ventilatory and heart rate responses to exercise: better predictors of heart failure mortality than peak oxygen consumption. Circulation 1999;100:2411-17.

\section{ELECTRONIC PAGES}

\section{eHEART: www.heartinl.com}

he following electronic only articles are published in conjunction with this issue of Heart.

\section{Catheter ablation of sinoatrial re-entry tachycardia in a 2 month old infant}

\section{T Simmers, N Sreeram, F Wittkampf}

Successful catheter ablation of sinoatrial re-entry tachycardia in an infant has not been previously reported. This procedure is described in a 2 month old boy with tachycardia induced cardiomyopathy.

(Heart 2003;89:el) www.heartjnl.com/cgi/content/full/89/1/el

\section{Sisters with atypical Fabry's disease with complete} atrioventricular block

Y Doi, G Toda, K Yano

A 56 year old woman with severe right heart failure and complete atrioventricular block was referred to hospital for further examination. Symptoms and signs suggestive of Fabry's disease, such as corneal opacities, acroparaesthesias, hypohidrosis, and angiokeratoma, were not noted. Echocardiography showed a diffuse hypertrophic left ventricular wall and paradoxical movement of the interventricular septum. Cardiac catheterisation showed restrictivetype ventricular dysfunction. Left ventricular endomyocardial biopsy showed central vacuolar degeneration of myocytes with inclusion bodies, which had a concentric lamellar configuration under electron microscopy. This finding is specific for Fabry's disease. The patient's elder sister had experienced an almost identical clinical course and histological findings of myocardial cells on necropsy. In conclusion, two sisters were encountered displaying interesting cases of atypical Fabry's disease. Symptoms began with complete atrioventricular block and histological myocardial findings were specific for Fabry's disease.

(Heart 2003;89:e2) www.heartjnl.com/cgi/content/full/89/1/e2 